\title{
Physical characterization methods for supplementary cementitious materials
}

\author{
Eleni C. Arvaniti - Maria C. G. Juenger - Susan A. Bernal • \\ Josée Duchesne $\cdot$ Luc Courard $\cdot$ Sophie Leroy $\cdot$ John L. Provis • \\ Agnieszka Klemm • Nele De Belie
}

Received: 2 May 2014/ Accepted: 26 September 2014/Published online: 4 October 2014

(C) RILEM 2014

\begin{abstract}
The main supplementary cementitious materials (SCMs) that are used today are industrial by-products. In most cases the quality of these materials cannot be controlled during their production, resulting in materials with varied characteristics. The adequate physical characterization of SCMs is important to better predict their performance and optimize their use in concretes production. There are standardized methods used to determine the particle characteristics for Portland cements that are usually adopted to characterize SCMs; however, these methods may not be as accurate when applied to SCMs. This paper is an overview of the techniques that are currently used for the determination of the density, particle size distribution, surface area and shape of SCMs. The main principles of each method are presented. The limitations that occur for the SCMs measurements are
\end{abstract}

\section{E. C. Arvaniti · N. De Belie ( $\square)$}

Magnel Laboratory for Concrete Research, Department of Structural Engineering, Faculty of Engineering and Architecture, Ghent University, Technologiepark Zwijnaarde 904, 9052 Ghent, Belgium e-mail: nele.debelie@ugent.be

\section{C. G. Juenger}

Department of Civil, Architectural and Environmental Engineering, University of Texas at Austin, 301 E. Dean Keeton St. C 1748, Austin, TX 78712, USA

\section{S. A. Bernal · J. L. Provis}

Department of Materials Science and Engineering, University of Sheffield, Sheffield S1 3JD, UK also discussed. This paper is an output from the work of the RILEM Technical Committee on Hydration and Microstructure of Concrete with Supplementary Cementitious Materials (TC-238-SCM).

Keywords Supplementary cementitious materials . Particle size measurement - Laser diffraction · Blaine . BET $\cdot$ Image analysis

\section{Introduction}

The use of supplementary cementitious materials (SCMs) in the production of concrete has increased worldwide over the past few decades [1, 2]. These materials can enhance the mechanical and durability

\author{
J. Duchesne \\ Département de géologie et de génie géologique, \\ Université Laval, Pavillon Adrien-Pouliot, \\ local 4507, 1065, ave de la Médecine, Québec, \\ QC G1V 0A6, Canada \\ L. Courard · S. Leroy \\ GeMMe Research Group, ArGEnCo Department, \\ University of Liege, Liege, Belgium
}
A. Klemm
Department of Construction and Surveying/School of Engineering and Built Environment, Glasgow Caledonian University, Cowcaddens Road, Glasgow G4 0BA, UK 
properties of concrete and contribute to mitigation of the environmental impact associated with the construction industry. SCMs are used as a partial replacement for Portland cement in concrete, reducing the fraction of Portland cement required to produce concrete with desired performance. Currently used SCMs are mostly by-products of industrial processes such as fly ashes derived from the coal-burning processes, blast furnace slags from the iron-making industry, and silica fume from ferro-silicon production [3]. However, in recent years, greater attention has been given to natural materials with pozzolanic activity such as calcined shales and clays, including metakaolin. Calcined clays and shales are used as cement replacements, while metakaolin is more often used as an additive to the cement.

The performance of SCMs in concrete is strongly dependent on their physical and chemical characteristics, which vary depending on the nature and source of the SCM. In general, the fineness is one of the most important physical properties controlling the reactivity of SCMs and the subsequent strength development of blended binders [4]. Reducing the average particle size increases the rate of dissolution of the SCM, raising the pozzolanic activity and thus the development of more strength-giving hydration products that enhance the long-term performance of the concrete. Small particles can also facilitate nucleation and growth of cement hydration products on the SCM surfaces, speeding up the early cement hydration and therefore the strength development. However, reducing the particle size of SCMs beyond an optimal value usually leads to an increased water demand of the concrete mixtures to achieve a desired workability, which can negatively affect both strength and its durability [5]. Further, if particle size is decreased by grinding, this requires additional energy costs.

For most industrial control purposes, the primary characteristics measured in powders are specific surface area, particle size distribution, particle shape, and density. The specific surface area (defined on a mass basis) is the most common property used to describe the fineness of Portland cement [6]. This surface area is an integral parameter and gives no information about details of the actual particle size distribution, which is probably of greater importance in defining concrete performance. The description of particle shape encompasses information about the sphericity and angularity, which affect workability and also the physical phenomena utilized for particlesize measurement [7]. Density can refer either to the unit volume of a packed powder, or to the specific gravity of the solid material itself. Both are key parameters in designing concrete, and particularly an accurate measurement of density is required for the conversion between volume and mass for calculation of the particle size distribution, and of the unit weight of concrete.

Even though SCMs are widely used by the construction industry, their physical characterization is challenging due to the varied characteristics typical of industrial by-products [8]. There are standardized methods used to determine particle characteristics of Portland cement that are usually adopted for the characterization of SCMs; however, these methods might not be sufficiently accurate for characterizing these materials. For instance, the air permeability test for specific surface area (Blaine), which is widely used for characterizing Portland cements [9], is based on the principle of resistance to air flow through a partially compacted sample of cement. This method relies on the assumptions that there is a relatively limited range of particle sizes in the material, with consistent inter-particle interactions, and that there are available, internationally accepted reference powders with properties similar to the material of interest. These conditions may not apply for all SCMs.

Deviations from expected results in physical characterization of powders are associated with instrument limitations, improper sample preparation procedures (e.g. inadequate dispersion), operator errors (e.g. improper instrument set-up or poor calibration), or incorrect sampling [10]. Although numerous techniques for the measurement of the physical characteristics of powders have been developed, most of the techniques are unsatisfactory in some respect, and there is no general method that may be applied with a reasonable confidence to a wide range of materials spanning several orders of magnitude in particle size, and with diverse particle shapes.

The key consideration for the proper and accurate determination of physical properties of SCMs lies in the selection of the adequate instruments and methods. This paper presents a critical overview of the techniques that are currently used for the determination of the particle size, specific surface area and shape of cementitious materials. The aim is to systematize the existing knowledge on this subject and to identify 
the most suitable techniques and methods that can be applied to characterize SCMs. This paper first discusses the methods of sample preparation prior to characterization, such as sampling and dispersion, and then describes the standard methods for testing density, specific surface area, particle size distribution, and particle shape.

\section{Methods for sample preparation}

\subsection{Sampling}

Sampling is a very important step in the characterization of any material and is one of the factors which can introduce the largest errors in particle size, shape, and density measurements of a powder [11]. Whenever a powder is analyzed, whether for physical or chemical assay, the quality of the measurement depends on how representative the sample is of the bulk from which it is drawn. The International Standard ISO 14488 [12], and the American standards ASTM C 183-08 [13] and ASTM C311/C311 M-13 [14], provide useful information on the requirements for sampling of finely divided materials.

Two types of sampling errors are possible [10]. First, statistical errors arising from sample heterogeneity cannot be prevented, but can rather be estimated beforehand and reduced by increasing the sample size. Even for an ideal random mixture, the quantitative particle distribution in samples of a given magnitude is not constant but is subject to random fluctuations. Second, there are errors that occur due to the segregation of the bulk and depend on the previous history of the powder. Dry powders tend to separate if they are stored for some time or they are vibrated during storage and transport.

There are several different techniques of sampling that have been evaluated in multiple studies (Table 1). The spinning riffle is the most reproducible method for obtaining a representative sample for powdered materials when compared with other techniques such as scoop sampling, table sampling, cone and quartering and chute riffling [10].

\subsection{Dispersion}

The term 'dispersion' has a variety of meanings, but in this context it makes reference to the process of
Table 1 Methods of powder sampling and associated error (data from [10] and [15])

\begin{tabular}{lll}
\hline Method & $\begin{array}{l}\text { Relative } \\
\text { standard } \\
\text { deviation }(\%)\end{array}$ & $\begin{array}{l}\text { Estimated } \\
\text { maximum sample } \\
\text { error }(\%)\end{array}$ \\
\hline Cone \& quartering & 6.81 & 22.70 \\
Scoop sampling & 5.14 & 17.10 \\
Table sampling & 2.09 & 7.00 \\
Chute riffling & 1.01 & 3.40 \\
Spin riffling & 0.125 & 0.42 \\
\hline
\end{tabular}

separating solid particles from each other to measure the physical characteristics of a given powder. For the characterization of SCMs, dispersion is particularly important in accurate determination of particle size distribution; however, if the natural agglomerated state is of interest, this should be taken into account during the sample preparation to avoid the break-up of agglomerated particles. In either case, the dispersion medium, whether air or liquid, should not cause irreversible changes to the particle size through processes such as dissolution, grinding or aggregation.

Dispersibility is defined as the ease with which a dry powder can be dispersed in a particular liquid medium, and depends on the lyophilicity, particle size, specific gravity and ionic charges on the surface (zetapotential) of the material [16]. Dispersibility is especially important when characterizing Portland cement and SCMs because there are situations (e.g. silica fume) when the particles are highly agglomerated in the dry state, and therefore must be properly dispersed in order to determine the "true" particle size distribution (PSD). There are no accepted standard methods for dispersing SCMs particles prior to analysis, and therefore the degree of dispersion will vary depending on the method that is used.

The main dispersion methods used for the cement industry include dry dispersion using high pressure air, and wet dispersion using solvents instead of water to minimize the risk of potential hydration reactions during testing. Wet dispersion usually involves sonication or ultrasonication to maximize the differentiation between weakly bounded agglomerates and the primary units. Factors such as particle size, geometry of the dispersing device, the residence time in the applied force and air pressure are some of the factors that can affect the degree of dispersion achieved. In the case of using wet methods for dispersing powdered particles, the surface 
chemistry of the powders, the solids concentration, and the amount of mechanical energy applied to break the agglomerates are the main factors that can induce variation in the dispersibility [17].

\subsection{Outgassing}

Outgassing involves the conditioning of the powder at a given temperature in helium or nitrogen flow. Outgassing strongly influences the measurement of specific surface area; testing partially moist particles covered with molecules of previously adsorbed gases or vapor, can lead to reduced or variable specific surface values [18]. There are no established outgassing conditions to assure accurate measurements of specific surface of powders. Most laboratories use their own standard protocol at an established temperature, gas pressure and time of outgassing, independent of the chemistry and structure of the material to test.

The ideal practice for determining the outgassing conditions should involve the study of the potential impact of different outgassing conditions on the physical and chemical properties of the tested material, to make sure that the original surface of the particles evaluated is preserved after this treatment. For most purposes, the outgassing temperature can be selected within the range where the thermogravimetric trace of the powder tested exhibits a minimum slope [19].

Outgassing can be conducted at room temperature $\left(20-25^{\circ} \mathrm{C}\right)$ when the powder is treated with a combined purge of a non-reactive, dry gas flow under vacuum, or when the specimens are subjected to desorption-adsorption cycles. These methods of outgassing are strongly recommended when analyzing materials that can suffer structural changes when exposed to elevated temperatures.

\section{Methods for physical characterization of SCMs}

Density, particle size distribution, shape and size are the fundamental physical characteristics of powders. The challenge in physical property characterization is to develop physical tests that can satisfactorily characterize key parameters. The techniques that are currently used for the characterization of cementitious materials include sieving, air permeability testing (Blaine), gas adsorption (BET), laser light scattering, and image analysis. Mercury intrusion porosimetry (MIP) for particle size analysis is also considered in this study, even though it is not yet a widely validated and used technique.

\subsection{Density}

The density of an SCM is employed in particle size analysis when there is a need to convert from volumetric particle size measurements to mass percentages of particles in a given size range. Density is also used in volumetric methods of concrete mixture design, such as that described in ACI 211.1 [20]; although Portland cement is usually replaced with an SCM on a mass percent basis, an accurate measurement of the volume of cementitious material is needed to correctly determine the proportions of the remaining components of the mixture. Density measurements on powders are generally conducted on the basis of volumetric displacement of a fluid. The presence of closed internal porosity in an SCM can introduce errors in the results of these tests, as the pores would not be accessible to the fluid, as in fly ashes with cenospheres and plerospheres. For SCMs with high internal porosity, the values obtained using different fluids in density tests may diverge depending on the accessibility of the internal pores to the fluid employed in testing.

For SCMs, standardized tests used for measuring density and/or specific gravity of Portland cements are generally adapted with little to no modification. For example, ASTM C 188 "Standard Test Method for Density of Hydraulic Cement" [21] measures density of cement using the volume of a liquid (kerosene or naptha) displaced by the powder in a Le Chatelier flask. ASTM C311 "Standard Test Methods for Sampling and Testing Fly Ash or Natural Pozzolans for Use in Portland-Cement Concrete" [22] modifies ASTM C188 for testing SCMs only by changing the mass of sample to be tested from (64 to $50 \mathrm{~g}$ ), to account for the lower density of these materials than Portland cement. Error can be introduced into this test method if the operator does not carefully follow the guidance with respect to temperature and material dispersion during testing. The latter may be difficult with some pozzolans.

Another option for measuring SCM density is using a gas pycnometer, following, for example, the procedure detailed in ASTM C604 "Standard Test Method for True Specific Gravity of Refractory Materials by 
Gas-Comparison Pycnometer" [23]. This method measures density by determining the volume of helium gas that can be introduced into a sample chamber of defined size which contains a known mass of powder; it is not modified for use with SCMs.

\subsection{Sieve analysis}

The simplest means of assessing the fineness of SCMs is through a sieve analysis since it does not require the specialized instrumentation used in other methods. However, the information obtained from sieve analysis of a fine powder is more limited than that obtained through more sophisticated methods, as the most commonly used sieve analysis procedures for powders utilize only one sieve size. For example, ASTM C618 [24] specifies a maximum of $34 \%$ by mass retained on the no. 325 sieve $(45 \mu \mathrm{m}$ opening size) for fly ash and natural pozzolans when wetsieved. Like density testing, the standard method used is one for Portland cement, ASTM C430 "Standard Test Method for Fineness of Hydraulic Cement by the 45- $\mu \mathrm{m}$ (No. 325) Sieve" [25]. The test standardizes the material mass to be tested, the water pressure and nozzle type, and sieve calibration procedures. As long as the material is in contact with water it does not react. Sources of error in this test can arise if the pozzolans are not adequately dispersed, as in densified silica fume for example; agglomerated particles will not pass the sieve opening under the low water pressure specified.

An alternative to the wet sieving process is to use a dry, forced-air process to sieve SCMs as described in EN 196-6 [26]. This process can be more rapid than a wet process since the sample does not need to be dried after testing. For example, Hooton and Buckingham [27] demonstrated that an air-jet sieve using forced air from a pressure-controlled vacuum measured the percent fly ash retained on the no. 325 sieve in approximately $2 \mathrm{~min}$. The values obtained are necessarily slightly different than those obtained using the wet-sieve process, tending to be slightly lower [28], but can be corrected using empirically-determined calibration factors.

\subsection{Air permeability test (Blaine)}

Air permeability methods measure the resistance of flow of air through a packed bed of cement of known dimensions and porosity. The time taken for a fixed quantity of air to flow through a compacted material bed of specified dimension and porosity is measured. Under standardized conditions, the specific surface of the material, commonly referred to as the Blaine fineness, is proportional to $\sqrt{ } t$, where $t$ is the time for a given quantity of air to flow through the compacted bed. The number and size range of individual pores in the specified bed are determined by the particle size distribution, which also influences the time for the specified air flow.

In 1939, Lea and Nurse introduced the constant flow-rate method that forms the basis of British Standard BS 4550 [28]. A simpler, constant volume method that is widely used in USA, UK and many other countries was developed by Niesel [29]. The apparatus (Fig. 1) consists of a U-tube manometer, a plunger, a permeability cell and a perforated disc. It is calibrated according to the Lea and Nurse method and the results are analyzed using the Carman-Kozeny equation [30] for viscous flow through a bed, which involves knowledge of the density of the cement (or of the SCM). However, this requires that the bed is uniform (which is very difficult to achieve for platyshaped particles such as those in most metakaolins), and that none of the particles are very highly irregular in shape (which is violated for fly ashes with punctured cenospheres and/or unburnt coal residues, or rice husk ashes retaining some of the geometry of the original plant material). In the case of silica fume it is impossible to form a compacted bed.

Two current standard testing methods are in widespread use for the analysis of Portland cement by this technique, the American standard ASTM C204-11 [31] and the European Standard EN 196-6 [26]. Both standards cover the determination of the fineness of hydraulic cement, in terms of the specific surface expressed as total surface area in square centimeters per gram of cement. For the calibration of the apparatus a trial quantity of $2.80 \mathrm{~g}$ of cement in ASTM C204-11 and 2.90 g of cement in EN 196-6 are taken. The apparatus constant in EN 196-6 is determined by measuring the permeability of the reference material of a known specific surface area.

Both standards take into account a compacted bed of the material formed in a special permeability cell to porosity $e=0.500 \pm 0.005$. Fine materials, though, other than cement may prove difficult to form into a compacted bed of porosity $e=0.500$. The reason for 


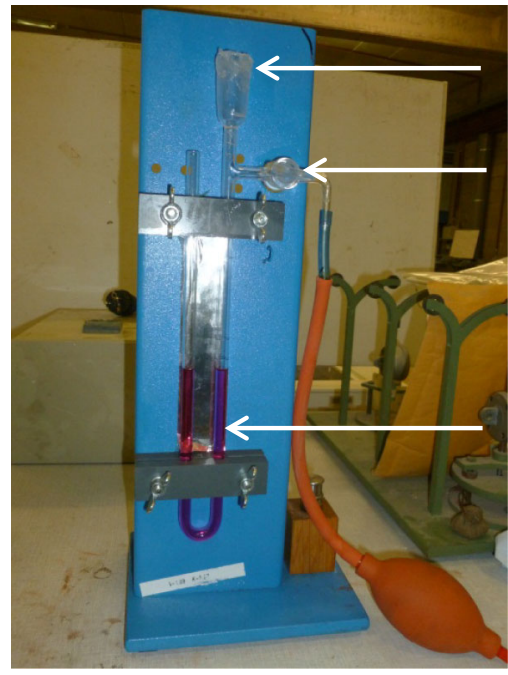

Standard taperfemale coupling to fit bottom of cell

Valve or clamp

Glass tube

Fig. 1 Blaine air permeability apparatus

this may lie in the fact that thumb pressure on the plunger cap may fail to bring it in contact with the top of the cell or, after making contact and removing the pressure, the plunger may move upwards as the bed restores semi-elastically to a larger volume. The porosity of $e=0.500$ is therefore likely to be unattainable for some materials. For such cases, the porosity required for a well-compacted bed needs to be determined experimentally. However, both standards consider equations for the calculation of fineness of materials other than cement. In these equations constants are also included which are appropriate for hydraulic cement.

The air permeability test is a simple and rapid method that is used in the cement industry [32]. However, air permeability is an indirect method and suffers from a number of weaknesses, including an inability to account for variable particle shape and bed tortuosity. A significant amount of the surface area of pores and cracks do not contribute to the flow resistance, and so a lower result than expected may be obtained.

The method is comparative rather than absolute and, therefore, a reference sample of known specific surface is required for calibration of the apparatus. The reference material must have similar shape, particle size distribution, and surface properties to the material of interest or it cannot be a valid comparison. In addition, the test is designed for cement, so it becomes extremely unreliable at surface areas greater than $500 \mathrm{~m}^{2} / \mathrm{kg}$ [32]. Its application to fly ash was suggested to be of debatable value because of the unknown extent of influence of the internal surface of unburned carbon particles present. Kiattikomol et al. [33] came to the conclusion that Blaine fineness may not be sufficient to indicate the fineness of fly ash, especially for fly ash with spongy phases. The full form of the Carman-Kozeny equation includes an explicit sphericity term, which is incorporated into the apparatus constant $K$ (EN 196-6) in the Blaine method, and so it is essential that the reference material is of a similar particle shape to the sample to be analyzed.

\subsection{Brunauer, Emmett and Teller (BET) surface area analysis}

In contrast to air permeability, the BET technique is a fundamental measurement of specific surface area because it makes no assumption about the shape of the particles. The BET method is based on the adsorption of a gas on the surface of the solid, including any surface pores and cracks that the gas molecules can access, and calculating the amount of adsorbed gas corresponding to a monomolecular layer on the surface. Nitrogen is the most commonly used gas, but any other inert gas can in principle be used. The physical adsorption of the gas results from Van der Waals forces between the gas molecules and the adsorbent surface area of the powder. The measurements are conducted at low temperature (often the boiling point $\left(-196{ }^{\circ} \mathrm{C}\right)$ of liquid nitrogen at atmospheric pressure, when $\mathrm{N}_{2}$ is the probe molecule) and the amount of gas adsorbed can be measured by a volumetric or continuous flow procedure.

Prior to BET analysis it is necessary to remove the gases and vapors that can be physically adsorbed on the surface of the particles. This procedure is known as outgassing (Sect. 2.3). It is important to bear in mind that BET analysis has some limitations, as the possibilities of micropore filling or penetration into cavities of molecular size are not considered in the measurement, which can generate false results. When characterizing a material it is recommended to measure at least three, but preferably five or more points, in the adequate pressure range on the $\mathrm{N}_{2}$ sorption isotherm, to obtain reliable results. The conditions of outgassing, the temperature of the measurements and the range of linearity of the BET plot should be reported along with the BET values [19]. 


\subsection{Laser diffraction}

Laser diffraction (LD) is rapidly becoming the most popular method for particle size determination [17]. Technology and instrument characteristics have rapidly developed in the last decades, and now LD is considered to be one of the quicker, easier and more reproducible methods of characterizing particle size, because it provides a complete picture of the full size distribution [34]. However, in laser diffraction the mathematical models used assume that the material is isotropic and consists of particles which can be approximated as spheres, meaning that the size of the particle is determined as the diameter of a spherical particle with an equivalent volume. These assumptions do not always hold for cements and SCMs, as will be discussed in more detail next.

The International Standard ISO 13320 [35] on Particle Size Analysis for Laser Diffraction Measurements is an introduction to laser diffraction particle sizing systems giving information on theory, guidance on both dispersion and sampling, and a methodology for proper quality control. However, the process by which a method can be validated is not clear from this document.

It should be made clear that laser diffraction instruments do not measure particle size distributions (PSD). What is measured is the light scattered by the particles. To relate this to the particle size distribution, critical assumptions are made about the optical properties of the material under analysis. A mathematical model is needed to convert light scattering data to particle size distribution. Two optical models are commonly used to calculate PSD, the Fraunhofer diffraction model and the Mie theory.

The Fraunhofer approximation assumes that: (1) the particle being measured is much larger than the wavelength of the light employed (ISO13320 defines this as being greater than $40 \lambda$, i.e. $25 \mu \mathrm{m}$ when a He$\mathrm{Ne}$ laser is used), (2) all sizes of particles scatter with equal efficiencies, and (3) the particles are opaque, transmitting no light. The Fraunhofer model does not make use of any knowledge of the optical properties of the sample, and only scattering at the contour of the particles (i.e. diffracted light) is considered to calculate the projected area of a sample. It is important to note that diffraction is independent of the composition of the particles [36], unlike the reflection and refraction that are not considered in this model.
Mie theory [37], on the other hand, is a more accepted theory used in LD measurements. The latest laser diffraction instruments use the full Mie theory, which completely solves the equations for interaction of light with matter (including diffraction, reflection and refraction of light). This can provide accurate results over a large size range (typically $0.02-2,000 \mu \mathrm{m})$, as long as the optical properties (refractive and absorption indices) of both the material and medium are known. The Mie theory determines the volume of the particle, as opposed to Fraunhofer model which predicts size based on a projected area. Whereas the Fraunhofer approximation is not suitable for samples that are transparent or semitransparent, and for small particles (i.e. less than $50 \mu \mathrm{m}$ ), Mie theory can apply under these conditions [35].

\subsubsection{Optical parameters}

In order to apply the Mie theory, the optical parameters of the tested particles are required. The optical properties determine how light interacts with a material, and are defined by the complex index of refraction, $\tilde{n}$ :

$\widetilde{n}=n-i k$

where $i=\sqrt{-1}$ and $n$ and $k$ are the real and the imaginary parts of the complex index of refraction. The real part, $n$, is called the refractive index while $k$ is the absorption (or extinction) coefficient. Both coefficients are dependent on the frequency of light and standard refractive index measurements $(n)$ are often tabulated for wavelengths emitted by a sodium flame or a sodium vapor lamp $(589.3 \mathrm{~nm})$ designated "D" [38]. The absorption coefficient ' $k$ ' is 0 or very close to 0 for transparent or translucent material, and becomes important for opaque media. In thin sections under the microscope a material will look opaque if its $k$-value (the absorption coefficient) is greater than 0.01 [39].

The real part of the refractive index of a liquid or a gas is easily measured using a suitable refractometer. However, the refractive index of a solid material in the form of a fine powder is more difficult to measure, due mainly to the fact that the material is often composed of more than one phase, which differ in their optical properties. In fact, only a limited number of materials have a single, isotopic refractive index: crystals belonging to the cubic crystal system, glasses and amorphous substances. Minerals in other crystallographic systems are anisotropic, with different 
refractive indices depending on crystal alignment with 2 or 3 main values described as $\alpha, \beta$ and $\gamma$ in tabulations of reference data $[38,40]$. The real part of the complex index of refraction can be determined under an optical microscope using the immersion method, in which the index of a solid is compared with that of a liquid of known index [9]. A material grain which is immersed in a liquid of matching refractive index as itself disappears from view. However, if the liquid is of a different refractive index the grain stands out, surrounded at the interface between the grain and the liquid by a thin band of light known as the Becke line [9]. The greater the difference in the refractive indices between the fragment and immersion, the greater is the intensity of the interface. By using a series of liquids of varying refractive indices, the refractive index of the material grain can be determined [9].

Direct measurement of the fundamental optical properties (refractive index $n$ and extinction coefficient $k$ ) can also be constructed using spectroscopic ellipsometry, which is an optical measurement technique that characterizes light polarization after reflection (or transmission) from a sample over a wide spectral range [41].

The use of correct input values for the optical parameters is very important for particle size determination of smaller particles. When particles are large and have a high refractive index difference compared to the suspension medium (e.g. air), and if the absorption is low [42], the errors resulting from incorrect input of these parameters are much smaller. Zhang and $\mathrm{Xu}$ [43] reported results on the effect of particle refractive index on size measurement and noted that it is well known from Mie theory that the scattered light differs for particles with different refractive indices, although the size or the distribution of the particles may be the same'. Their conclusion was that if an incorrect refractive index is assumed when the particle size distribution is computed from a measured scattered energy distribution, a $10 \%$ error will be involved under most circumstances, but greater errors may occur if the assumed refractive index is much less or greater than the actual one.

In the case of Portland cement, the refractive index is not a single value since cement is a multiphase powder. Mean values are often calculated based on the known optical properties for each constituent pure phase [44]. According to Hackley et al. [42], for cementitious powders, absorption becomes important for the fine fraction, below about $1 \mu \mathrm{m}$ in diameter, where it can have a large impact on the particle size distribution. For $n \geq 1.6$ (fairly refractive materials), the model is not very sensitive to the choice of $n$ for weakly absorbing or transparent materials (i.e., $k<0.1)$. It is only moderately sensitive at $k=0.1$. The magnitude of the calculated submicron fraction depends on the choice of $k$, with the dependence being stronger as $n$ becomes smaller [42].

Table 2 presents refractive indices $n$ values for phases often present in Portland cement and SCMs.

Values for the absorption coefficient $k$ are less frequently reported. For cements the imaginary parts $k$ of the complex refractive index are reported within the very wide interval of $k=0.003$ to 1.0 [47]. A mean value of 0.1 was used by Hooton and Buckingham [27] for ordinary Portland cement and fly ash, and 0.001 for silica fume. Gupta and Wall [48] presented a range for $k$ from 0.005 to 0.01 for char-free ash from subbituminous coal, while values between 0.005 and 0.05 have also been reported $[49,50]$. However, those authors (working on the radiative properties of fly ashes) concluded that it is not acceptable to ignore the wavelength-dependence of fly ash refractive index, and that previous studies employing $n=1.5$ and $k$ ranging from 0.005 to 0.05 seem to overestimate the Planck mean absorption coefficient of fly ash particles. Liu and Swithenbank [50] mentioned that the average value of the imaginary part of the fly ash complex refractive index $k=0.012$ estimated by Gupta and Wall [51] is definitely too high.

Reported values in the literature for the refractive index and the absorption coefficient of ordinary Portland cement and SCMs are shown in Table 3.

\subsection{Particle size analysis by microscopy image analysis}

Image-based particle size analysis relies on the principle developed by Medalia [54]. Modern image analysis uses scientific cameras to provide low distortion digital images that are instantaneously processed to extract particle size and shape. The imaging setups have to be calibrated in terms of illumination and spatial resolution. Illumination is usually adjusted through trial and error procedures to optimize the contrast between the background and the objects to be measured. A blank image consisting in the imaging of the single background is usually acquired to allow 
Table 2 Typical refractive index values ( $\mathrm{n}$ ) for phases often present in cements and SCMs

\begin{tabular}{llllll}
\hline Phase & $n$ & Ref & Phase & $n$ & Ref \\
\hline Pure $\mathrm{C}_{3} \mathrm{~S}$ & $1.7139-1.07238$ & {$[45]$} & $\beta-\mathrm{C}_{2} \mathrm{~S}$ & $1.717-1.735$ & {$[45]$} \\
$\mathrm{C}_{4} \mathrm{AF}$ & $1.96-2.04$ & {$[45]$} & $\gamma-\mathrm{C}_{2} \mathrm{~S}$ & $1.642-1.654$ & {$[45]$} \\
Arcanite & $1.4935-1.4973$ & {$[45]$} & $\mathrm{Ca}(\mathrm{OH})_{2}$ & $1.545-1.573$ & {$[45]$} \\
Gypsum & $1.5205-1.5296$ & {$[45]$} & Hemihydrate & $1.559-1.5836$ & {$[45]$} \\
$\gamma-$ CaSO $_{4}$ & $1.505-1.548$ & {$[45]$} & Syngenite & $1.5011-1.5176$ & {$[45]$} \\
MgO & 1.736 & {$[40]$} & Gehlenite & $1.658-1.655$ & {$[40]$} \\
Akermanite & $1.632-1.64$ & {$[40]$} & C-S-H & $1.49-1.530$ & {$[40]$} \\
Hydrogarnet & $1.604-1.734$ & {$[40]$} & Merwinite & $1.708-1.724$ & {$[40]$} \\
Mirabilite & $1.394-1.398$ & {$[40]$} & Thenardite & $1.464-1.485$ & {$[40]$} \\
Ettringite & $1.462-1.466$ & {$[40]$} & Monosulfate & $1.488-1.504$ & {$[40]$} \\
Thaumasite & $1.468-1.504$ & {$[40]$} & Quartz & $1.544-1.553$ & {$[40]$} \\
Calcite & $1.486-1.658$ & {$[40]$} & Mullite & $1.642-1.654$ & {$[40]$} \\
Magnetite & 2.42 & {$[46]$} & Maghemite & 2.54 & {$[46]$} \\
Hematite & $2.87-3.22$ & {$[46]$} & Cristobalite & $1.485-1.487$ & {$[46]$} \\
Rutile & $2.605-2.908$ & {$[46]$} & Anatase & $2.488-2.561$ & {$[46]$} \\
\hline
\end{tabular}

Table 3 Typical refractive index values (real) and absorption index values (imaginary) of cement and cementitious materials

\begin{tabular}{llll}
\hline Material & $\begin{array}{l}\text { Refractive } \\
\text { Index, } n\end{array}$ & $\begin{array}{l}\text { Absorption } \\
\text { coefficient, } k\end{array}$ & Reference \\
\hline OPC & 1.73 & 0.1 & {$[52]$} \\
Fly ash & 1.73 & 0.1 & {$[52]$} \\
& 1.50 & $0.005-0.05$ & {$[50]$} \\
Fly ash, Class F & 1.56 & 1.0 & {$[53]$} \\
Fly ash, Class C & 1.65 & 0.1 & {$[53]$} \\
BFS, GFS & 1.62 & 1.0 & {$[53]$} \\
Silica fume & 1.53 & 0.001 & {$[52]$} \\
\hline
\end{tabular}

$O P C$ ordinary Portland cement, $B F S$ blast furnace slag, $G F S$ gasification slag

spatial correction of the illumination of the field of view.

The illumination intensity also needs to fit to the camera sensitivity to guarantee the shorter exposure time possible. Camera saturation should, however, always be avoided, as it can cause some blurring effect and compromise accurate measurement. Various illumination geometries can be envisaged; however, the highest resolution is achieved with axial back-lighting of the particles [55, 56]. Once the illumination setup is fixed, the spatial resolution in the field of view has to be calibrated. This is usually performed by imaging a reference grate whose size is well-known.
Overall imaging setups are commonly classified into two main categories: dynamic or static. In dynamic image analysis setups, particles are moving or free-falling in the field of view of the camera which induces some uncertainties related to the particle position and orientation. The impact on the measurement is particularly sensible in the case of elongated particles. In the static setups, particles are at rest in a plane, thus the probability to measure the particle longest dimensions is statistically high. The more controlled static setups should be preferred for accurate measurements [57].

\subsection{Mercury intrusion porosimetry (MIP) for PSD}

MIP has formed the basis for a number of internationally recognised standard analyses; however it seems, as observed by León [58], that the full extent of its capabilities remains underexploited.

Generally MIP is used to study the volume, distribution and interconnectivity of the voids (pores) within porous solid and fine-grained samples, relying on the Washburn equation to build a picture of the microstructure. On the premise that for a given pore radius, a certain pressure is required to intrude a nonwetting fluid (mercury) and by recording the volume intruded for each pressure increment, an intrusion curve may be derived. It can be represented by the Washburn formula: 
$P=-2 \gamma \cos (\theta) / r$

where $P$ is the pressure, $\gamma$ is the surface tension of the fluid, $\theta$ is the contact angle between the fluid and the material's surface, and $r$ is the pore radius [58, 59].

The Washburn theorem assumes a model of cylindrical pores, and the MIP technique further assumes that these cylinders get progressively smaller towards the inside of the sample. As pressure increases leading to further intrusion, it is assumed that this is attributable to progressively smaller pore radii [60]. Naturally, these assumptions are seldom representative of a porous material used in practice; however, the data produced when compared to data from other materials also assessed by MIP, can be valuable for depicting trends and interrelationships.

There is little information in the literature about particle size distribution determined via MIP [58, 61, 62]. Particle size distribution (PSD) by MIP is derived from Mayer and Stowe's [63] relationship established between particle size and breakthrough pressure required to fill the interstitial voids between a packed bed of spheres. Following the development of PSD by MIP, Mayer and Stowe presented the benefits of the spherical model for characterising certain types of porous solids, over that of the cylindrical model [64].

Employing the same principle of interfacial tensions giving resistant force to an intruding, nonwetting liquid, a curve of intrusion versus applied pressure is produced. PSD by MIP relies on the premise that this curve contains structural information about the studied material. MIP uses a model of cylinders for 'typical' pore analyses and a model of spheres for particle size analyses. The particle size distribution as analysed by the MIP curve is perhaps better termed the "Equivalent Spherical Size Distribution", as the calculated PSD derives from the modelled set of spheres which best represents the logged experimental data [65].

The question of how representative these results are is therefore dependent on how similar the particle geometry is to that of a set of spheres. Plate-like or very angular particles, such as in metakaolin and granulated blast furnace slag, conform less well to the mathematical model than mono-sized well-rounded particles, and therefore its applicability is less intuitively obvious.

This application of the MIP technique is subject to some criticism arising from, amongst other aspects, the set of inherent assumptions it relies on. Particle size distribution is an extension of many of these assumptions, including its own additional ones, and as such must be undertaken using a considered approach. The results of the technique display an approximation of the particle size distribution, rather than a measurement, and provide a 'feel' for the characteristics of the particles, used along with complementary techniques and viewed in an objective context.

Whilst Huggett et al. [61] considered the assumptions of Mayer-Stowe PSD by MIP to be "gross", seeking to refine the method and presenting their modified alternative approach, they did indeed find that the Mayer-Stowe technique presented "a good approximation" of PSD for certain types of samples.

Practically speaking, however crude the mathematical model may be when compared to the true sample, careful management of certain variables can hold significant benefit to the overall representativity of the analysis. One such consideration is that of fine pressure increments as a pragmatic way of increasing accuracy. Another is the contact angle assumed for analysis; setting a control contact angle (e.g. [66]), if perhaps a little crude, remains useful for comparing data from physically and chemically similar materials analyzed under the same technique [58].

\section{Conclusions}

The shape and size peculiarities of SCMs differentiate them from Portland cement, and therefore the techniques that are currently used for the physical characterization of cement may not be directly applicable to SCMs, in their standardized forms. Considering particle size distribution as one of the most important parameters for the optimization of SCM utilization, several techniques that are used for its determination have been reviewed in this study. Some of them are more widely used in Portland cement characterization, such as air permeability testing, sieving, laser diffraction, BET, and image analysis. It is elucidated that higher variability in the results is introduced through lack of validated sample pre-conditioning or testing protocols for characterizing SCMs. MIP is not generally applied to measure particle size distributions, but seems to be a promising technique for the PSD determination of mainly spherical-shaped SCMs. 


\section{References}

1. Lothenbach B, Scrivener K, Hooton RD (2011) Supplementary cementitious materials. Cem Concr Res 41(3):217-229

2. Snellings R, Mertens G, Elsen J (2012) Supplementary cementitious materials. Rev Mineral Geochem 74:211-278

3. Siddique R, Khan MI (2011) Supplementary cementing materials. Springer, Berlin

4. Celik IB (2009) The effects of particle size distribution and surface area upon cement strength development. Powder Technol 188(3):272-276

5. Scrivener KL, Kirkpatrick RJ (2008) Innovation in use and research on cementitious material. Cem Concr Res 38(2): $128-136$

6. Stark U, Mueller A (3003) Particle size distribution of cements and mineral admixtures-standard and sophisticated measurements. In: Owens DGGaG (ed) 11th International Congress on the Chemistry of Cement (ICCC), Durban, 11-16 May 2003

7. Naito M, Hayakawa O, Nakahira K, Mori H, Tsubaki J (1998) Effect of particle shape on the particle size distribution measured with commercial equipment. Powder Technol 100:52-60

8. Juenger M, Provis JL, Elsen J, Matthes W, Hooton RD, Duchesne J, Courard L, He H, Michel F, Snellings R, Belie ND (2012) Supplementary Cementitious Materials for Concrete: Characterization Needs. MRS Proceedings 1488

9. Hewlett P (2003) Lea's chemistry of cement and concrete, 4th edn. Butterworth Heinemann, Oxford

10. Allen T (1997) Particle size measurement, vol 1, 5th edn. Chapman and Hall, London

11. Allen T (2003) Powder sampling and particle size determination, 1st edn. Elsevier B.V, Heidelberg

12. ISO 14488:2007 (2007) Particulate materials-sampling and sample splitting for the determination of particulate properties

13. ASTM C 183-08 (2008) Standard practice for sampling and the amount of testing of hydraulic cement

14. ASTM C311/C311M-13 (2013) Standard test methods for sampling and testing fly ash or natural pozzolans for use in portland-cement concrete

15. Green DW (1997) Perry's chemical engineers' handbook, 7th edn. McGraw-Hill, London

16. Takeo Mitsui BS, Susumu Takada BS (1969) On Factors influencing dispersibility and wettability of powder in water. J Soc Cosmetic Chemists 20:335-351

17. Ferraris CF, Hackley VA, Aviles AI, Charles E. Buchanan J (2002) Analysis of the ASTM Round-Robin test on particle size distribution of portland cement: phase I. NISTIR 6883

18. Clausen L, Fabricius I (2000) BET Measurements: outgassing of minerals. J Colloid Interface Sci 227(1):7-15

19. Pierotti R, Rouquerol J (1985) Reporting physisorption data for gas/solid systems with special reference to the determination of surface area and porosity. Pure Appl Chem 57(4):603-619

20. ACI 211.1-91 (2009) Standard practice for selecting proportions for normal, heavyweight and mass concrete

21. ASTM C188-09 (2009) Standard test method for density of hydraulic cement
22. ASTM C311/C311M-13 (2013) Standard test methods for sampling and testing fly ash or natural pozzolans for use in portland-cement concrete

23. ASTM C604-02R12 (2012) Standard test method for true specific gravity of refractory materials by gas-comparison pycnometer

24. ASTM C618-12a (2012) Standard specification for coal fly ash and raw or calcined natural pozzolan for use in concrete

25. ASTM C430-08 (2008) Fineness of hydraulic cement by the 45- $\mu \mathrm{m}$ (No. 325) Sieve

26. EN 196-6 (2010) Methods of testing cement-Part 6: determination of fineness

27. Hooton RD, Buckingham JHP (1985) The use and standardization of rapid test methods for measuring the carbon content and fineness of fly ash pozzolan. In: 7th international ash utilization symposium, Orlando, FL, 4-7 March. Proceedings of the 7th International Ash Utilization Symposium

28. BS 4550:1978 (1978) Methods of testing cements

29. Niesel K (1973) Determination of the specific surface by measurement of permeability. Mater Struct 6(3):227-231

30. Schulz NF (1974) Measurement of surface areas by permeametry. Int J Miner Process 1(1):65-79

31. ASTM C204-11 (2011) Standard test methods for fineness of hydraulic cement by air-permeability apparatus

32. Potgieter JH, Strydom CA (1996) An investigation into the correlation between different surface area determination techniques applied to various limestone-related compounds. Cem Concr Res 26(11):1613-1617

33. Kiattikomol K, Jaturapitakkul C, Songpiriyakij S, Chutubtim S (2001) A study of ground coarse fly ashes with different finenesses from various sources as pozzolanic materials. Cem Concr Compos 23(4-5):335-343

34. Frías M, Sánchez de Rojas MI, Luxán Md, García N (1991) Determination of specific surface area by the laser diffraction technique. Comparison with the Blaine permeability method. Cem Concr Res 21(5):709-717

35. ISO 13320:2009 (2009) Particle size analysis-laser diffraction methods. Part I: general principles

36. Ferraris CF, Hackley VA, Aviles AI (2004) Measurement of particle size distribution in Portland cement powder: Analysis of ASTM round robin studies. J Cem Concr Aggreg 26(2):1-11

37. Wriedt T (1998) A review of elastic light scattering theories. Part Part Syst Charact 15:67-74

38. Wahlstrom EE (1979) Optical Crystallography, 5th edn. Wiley, New York

39. Mitra S (1996) Fundamentals of optical, spectroscopic and X-Ray mineralogy. New Age International, New Delhi, p 336

40. Glossary of Minerals. http://freeit.free.fr/Knovel/Concrete\% 20Petrography $\% 20-\% 20$ A $\% 20$ Handbook\%20of\%20Investi gative\%20Techniques/92669_gl

41. Fujiwara H (2007) Spectroscopic ellipsometry principles and applications. Wiley, London

42. Hackley VA, Lum LS, Gintautas V, Ferraris CF (2004) Particle size analysis by laser diffraction spectrometry: application to cementitious powders. Report from the US Department of Commerce. National Institute of Standards and Technology (NIST), NISTIR 7097 
43. Zhang HJ, Xu GD (1992) The effect of particle refractive index on size measurement. Powder Technol 70:189-192

44. Ferraris CF, Bullard JW, Hackley V (2006) Particle size distribution by laser diffraction spectrometry: application to cementitious powders. In: Proceedings of the 5th world congress on particle technology, Orlando

45. Taylor HFW (ed) (1997) Cement chemistry. 2nd edn. Telford, London

46. www.webmineral.com

47. Mykhaylo P, Gorsky PPM, Maksimyak AP (2010) Optical correlation technique for cement particle size measurements. Optica Applicata XL 40(2):459

48. Gupta RP, Wall TF (1985) The optical properties of fly ash in coal fired furnaces. Combust Flame 61:145-151

49. Goodwin DG, Mitchner M (1989) Flyash radiative properties and effects on radiative heat transfer in coal-fired systems. Int J Heat Mass Transfer 32(4):627-638

50. Liu F, Swithenbank J (1993) The effects of particle size distribution and refractive index on fly-ash radiative properties using a simplified approach. Int J Heat Mass Transf 36(7):1905-1912

51. Gupta RP, Wall TF (1981) The complex refractive index of particles. J Phys D Appl Phys 14:95-98

52. Cyr M, Tagnit-Hamou A (2001) Particle size distribution of fine powders by LASER diffraction spectrometry. Case of cementitious materials. Mater Struct 34(6):342-350

53. Jewell RB, Rathbone RF (2009) Optical properties of coal combustion byproducts for particle-size analysis by laser diffraction. Combustion and Gasification Products 1:1-6

54. Medalia A (1970) Dynamic shape factors of particles. Powder Technol 4:117-138

55. Leroy S, Dislaire G, Bastin D, Pirard E (2011) Optical analysis of particle size and chromite liberation from pulp samples of a UG2 ore regrinding circuit. Miner Eng 24:1340-1347
56. Gregoire M, Dislaire G, Pirard E (2007) Accuracy of size distributions obtained from single particle static digital image analysis. Partec, Nurnberg

57. Hart JR, Zhu Y, Pirard E (2009) Advances in the characterization of industrial minerals. Ch 4: Particle size and shape characterization: current technology and practice

58. León CA (1998) New perspectives in mercury porosimetry. Adv Colloid Interface Sci 76-77:341-372

59. Glass HJ, With G (1996) Reliability and reproducability of mercury intrusion porosimetry. $\mathrm{J}$ Eur Ceram Soc 17:753-757

60. Giesche H (2006) Mercury porosimetry: a general (practical) overview. Part Part Syst Charact 23:9-19

61. Huggett S, Mathews P, Matthews T (1999) Estimating particle size distributions from a network model of porous media. Powder Technol 104:169-179

62. Lucarelli L (2013) The use of mercury intrusion porosimetry for the determination of particle size distribution on nano-particles carbon black. http://s3.ceelantech.com/docs/ MercuryIntrusionPorosimetry.pdf. Accessed 21 Oct 2013

63. Mayer RP, Stowe RA (1965) J Colloid Sci 20:893-911

64. Mayer RP, Stowe RA (2005) Packed uniform sphere model for solids: interstitial access opening sizes and pressure deficiencies for wetting liquids with comparison to reported experimental results. J Colloid Interface Sci 294:139-150

65. Webb PA (2001) An introduction to the physical characterization of materials by mercury intrusion porosimetry with emphasis on reduction and presentation of experimental data. Micromeritics Instrument Corporation, Norcross

66. Drake LC, Ritter HL (1945) Pressure porosimeter and determination of complete macropore-size distributions. Ind Eng Chem Anal Ed 17:782-786 